\title{
Neonatal Enterovirus Infection: Case Series of Clinical Sepsis and Positive Cerebrospinal Fluid Polymerase Chain Reaction Test with Myocarditis and Cerebral White Matter Injury Complications
}

\author{
Frank H. Morriss Jr., MD, MPH ${ }^{1,2}$ Julie B. Lindower, MD, MPH ${ }^{1,2}$ Heather L. Bartlett, MD ${ }^{3}$
}

Dianne L. Atkins, $\mathrm{MD}^{1,2}$ Jean O. Kim, MD ${ }^{4}$ Jonathan M. Klein, MD ${ }^{1,2}$ Bradley A. Ford, MD, PhD ${ }^{2,5}$

${ }^{1}$ Stead Family Department of Pediatrics, University of lowa Children's

Address for correspondence Frank H. Morriss, Jr., MD, MPH,

Hospital, lowa City, lowa

2 Departments of Pediatrics and of Pathology, Roy J. and Lucille A. Department of Pediatrics, University of lowa Children's Hospital, 200 Hawkins Drive, lowa City, IA 52242

Carver College of Medicine, University of lowa, lowa City, lowa (e-mail: frank-morriss@uiowa.edu).

${ }^{3}$ Department of Pediatrics, University of Wisconsin, Madison,

Wisconsin

${ }^{4}$ Department of Pediatrics, Chicago Medical School, Rosalind Franklin University of Medicine and Science, North Chicago, Illinois

${ }^{5}$ Department of Pathology, University of lowa Hospitals and Clinics, lowa City, lowa

Am J Perinatol Rep 2016;6:e344-e351.

\begin{abstract}
Keywords

- enterovirus

- immunoglobulin intravenous

- meningoencephalitis

- myocarditis

- neonate

- periventricular leukomalacia

Objective We describe five neonates with enteroviral (EV) infection to demonstrate central nervous system (CNS) and cardiac complications and report successful treatment of myocarditis with immunoglobulin intravenous (IVIG) in two.

Study Design Case series identified during three enteroviral seasons in one neonatal intensive care unit (NICU) by cerebral spinal fluid (CSF) reverse transcriptase polymerase chain reaction ( $P C R$ ) testing for $E V$ in neonates suspected to have sepsis, but with sterile bacterial cultures.

Results Cases were identified in each of three sequential years in a NICU with 800 to 900 admissions/year. Two cases were likely acquired perinatally; all were symptomatic with lethargy and poor feeding by age 5 to 10 days. All had signs of sepsis and/or meningitis; one progressed to periventricular leukomalacia and encephalomalacia. Two recovered from myocarditis after treatment that included IVIG 3 to $5 \mathrm{~g} / \mathrm{kg}$.

Conclusion Neonates who appear septic without bacterial etiology may have EV CNS infections that can be diagnosed rapidly by CSF PCR testing. Cases may be underdiagnosed in the early neonatal period if specific testing is not performed. Neonates with EV infection should be investigated for evidence of periventricular leukomalacia, screened for myocarditis, and considered for IVIG treatment.
\end{abstract}

Suspicion for neonatal sepsis and/or meningitis prompts evaluations in as many as 69 to $82 \%$ of preterm infants $\leq$ 3 days age and 6 to $50 \% \geq 3$ days age, varying with gestational age. However, less than $1 \%$ of evaluations identify a bacterial or fungal etiology at $\leq 3$ days and less than $12 \%$ at $\geq 3$ days. ${ }^{1,2}$ Viruses also cause sepsis and meningitis in neonates, but are less commonly identified etiologies, in part because viral cultures and specific diagnostic tests are not consistently received

February 7, 2014 accepted after revision April 10, 2014
DOI http://dx.doi.org/ 10.1055/s-0036-1593406. ISSN 2157-6998.
Copyright $\odot 2016$ by Thieme Medical Publishers, Inc., 333 Seventh Avenue, New York, NY 10001, USA. Tel: +1(212) 584-4662.
License terms

(요 $\Theta \circledast$ 
performed. ${ }^{3}$ Specific diagnosis of neonatal enterovirus (EV) illness is important because of the need to screen for and treat complications and for infection control.

Included among the family Picornaviridae genus Enterovirus are viruses formerly identified as Coxsackieviruses, echoviruses, numbered enteroviruses, and polioviruses. ${ }^{4} \mathrm{EV}$ that cause illness in humans were reclassified in 2013 as Enteroviruses A, B, C, and $D$ on the basis of genetic similarity. The three poliovirus serotypes now belong to the species Enterovirus C. Human Parechoviruses, which belong to the same family of Picornaviridae as $\mathrm{EV}$, were until 1999 classified as enteroviruses.

Nonpolio EV have been recognized as the most common etiology of meningitis in infants ${ }^{5}$ and as a cause of neonatal myocarditis. ${ }^{6,7}$ The acquisition of the virus and the presentation of the broad spectrum of illness in neonates have been described in epidemiologic studies, ${ }^{7,8}$ case series, ${ }^{9}$ and reviews. ${ }^{10-12}$ However, perhaps because of the seasonal prevalence of EV and delay in reporting viral culture results, in clinical practice these viruses are not consistently considered in the evaluation of neonates with suspected sepsis and/or meningitis who have negative bacterial cultures.

We present five neonates with EV clinical sepsis without bacterial etiology who were diagnosed during August to October between 2011 to 2013 on the basis of a positive result of a cerebrospinal fluid (CSF) reverse transcriptase polymerase chain reaction (PCR) test for EV (Xpert EV Assay, Cepheid, Sunnyvale, $\mathrm{CA}$ ) after that test became available in our hospital in 2011. ${ }^{13}$ Each patient had evidence of EV RNA in CSF, but not all had CSF pleocytosis or abnormal neuroimaging. In a neonatal intensive care unit (NICU) with 800 to 900 annual admissions, these five cases over three nonepidemic EV seasons suggested to us that $\mathrm{EV}$ is a more common etiology of neonatal sepsis and/or meningitis than previously recognized and prompted an inquiry regarding the incidence of EV illness in neonates. As their hospital courses evolved, two patients developed myocarditis and recovered after aggressive immunoglobulin intravenous (IVIG) therapy, an outcome different from those reported in published EV myocarditis case series when unspecified IVIG doses were administered. Moreover, in the absence of a history of intraventricular hemorrhage or hypoxic-ischemic insult, one patient developed periventricular leucomalacia (PVL) that progressed to encephalomalacia and associated developmental delay, adding to the reported cases of this etiology of PVL.

\section{Cases}

The characteristics of the patients are presented in the - Table 1. Two were males. All were born during the late summer or fall. One was term, one was early preterm and three were late preterm, that is, 34 to $<37$ week gestational age. Birth weights were 1.7 to $3.7 \mathrm{~kg}$. Apgar scores were robust. One was born to a mother with a febrile illness associated with severe pleurodynia and chorioamnionitis that was thought to be of viral etiology; another was born to a mother with a prepartum respiratory tract infection; and a third was a water birth at home, where a sibling had very recently had diarrhea. Of four born in hospital, only the $<34$ week gestational age infant required an initial hospitalization $>2$ days, and three had been home prior to readmission. All presented with poor feeding and lethargy by age 10 days. Two were hypothermic and one was febrile. Only one developed an exanthem, none had a seizure and none developed hepatitis.

Serum C-reactive protein was increased in all patients during the acute illness. White blood count was abnormal in only one neonate whose value was low. Two infants had transient thrombocytopenia. CSF examination was remarkable for minimal or no pleocytosis, but all samples were positive for EV nucleic acid as tested by PCR assay. ${ }^{13}$ CSF PCR for herpes simplex virus (HSV) was negative in four, and CSF HSV cultures were sterile in the fifth. Bacterial cultures of blood and CSF in all and urine in three were sterile. No other sample types (i.e., stool, throat swabs, blood) were tested with the PCR for EV test, nor were they cultured for viruses.

With supportive therapy, each neonate gradually improved and all survived. However, two were diagnosed with myocarditis by age 16 to 24 days; one of these had been treated for hypotension beginning at age 7 days. Electrocardiographic telemetry demonstrated ventricular ectopy, which prompted further cardiac evaluation in this patient and the subsequent ones. Echocardiograms in two patients revealed decreased ventricular function. These two patients were treated with IVIG at 3 to $5 \mathrm{~g} / \mathrm{kg}$ total, milrinone, $\beta$ blockers, and digoxin, with clinical and echocardiographic improvement. One patient who did not have myocarditis was treated prophylactically with IVIG $0.75 \mathrm{~g} / \mathrm{kg}$ total.

The preterm infant who was treated for hypotension beginning at age 7 days and who subsequently was diagnosed to have myocarditis was an outborn infant who had good Apgar scores and was treated for respiratory distress syndrome before transfer to our institution. At age 16 days the patient had a normal head ultrasound, but by age 23 days, the head ultrasound examination revealed early changes of PVL, which by age 3 months progressed to encephalomalacia. This patient was developmentally delayed at age 9 months.

During the 25 months when these 5 neonates were admitted, NICU physicians elected to obtain a CSF EV PCR test on a total 26 patients; the test was negative in 21 .

\section{Discussion}

Incidence of neonatal $\mathrm{EV}$ infections: These five patients with $\mathrm{EV}$ infection, who were diagnosed during three $\mathrm{EV}$ seasons in one NICU using a newly available CSF PCR test for EV, led us to examine the discrepancy between the relatively low identification of $\mathrm{EV}$ as a cause of neonatal illness in clinical practice and the more frequent identification of neonatal $\mathrm{EV}$ infection when it is more rigorously sought.

Two prospective epidemiologic studies demonstrated relatively high incidences of neonatal sepsis of viral etiology. In one, a 13-month Finnish population-based prospective survey of 137 late preterm and term newborn infants with suspected systemic infection in which viral cultures were obtained in all, 4 (3\%) had positive bacterial blood cultures and $11(8 \%)$ had adenovirus or EV infections proven by culture of blood $(n=7)$ and/or feces $(n=4) .^{3}$ In the second study, a 


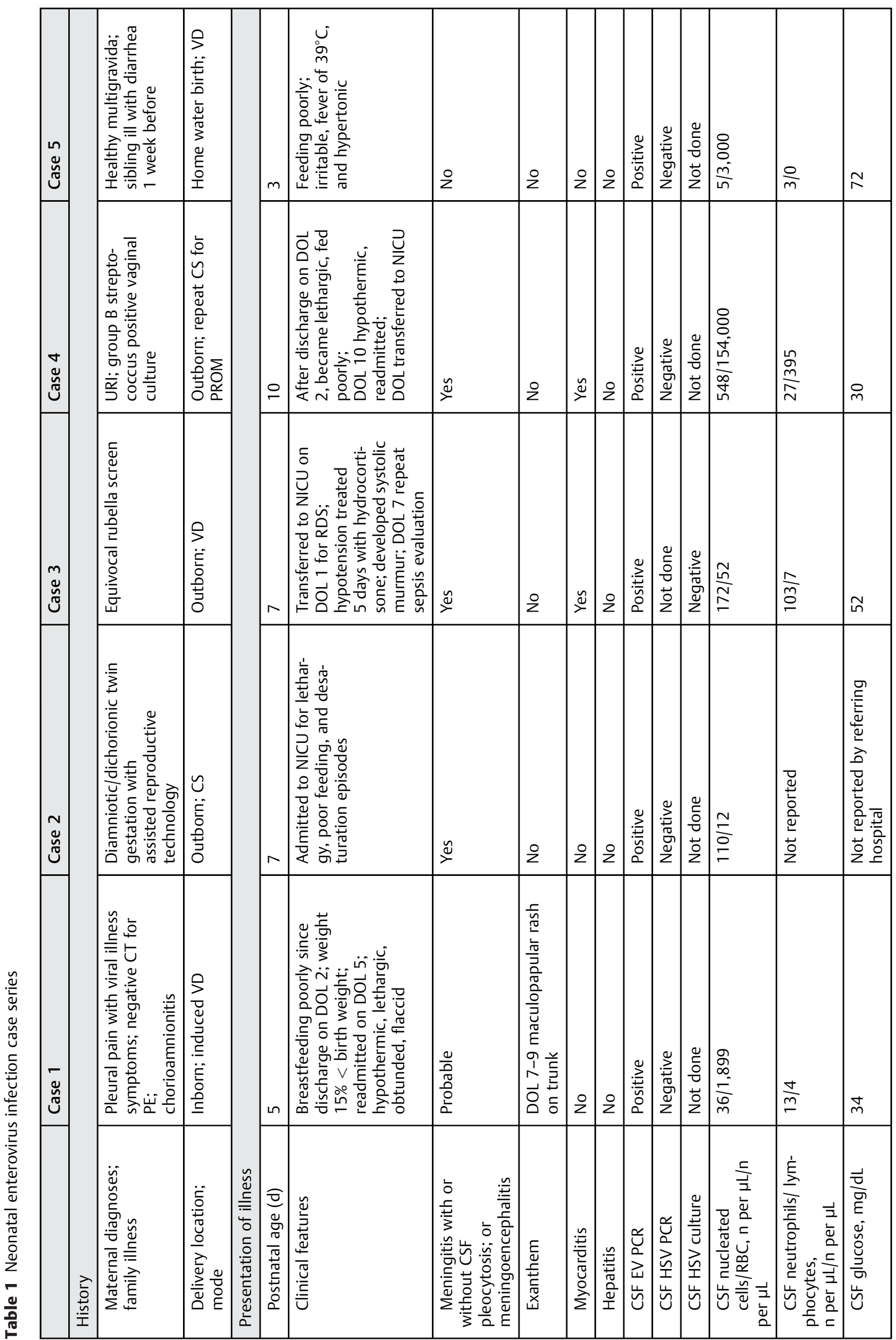




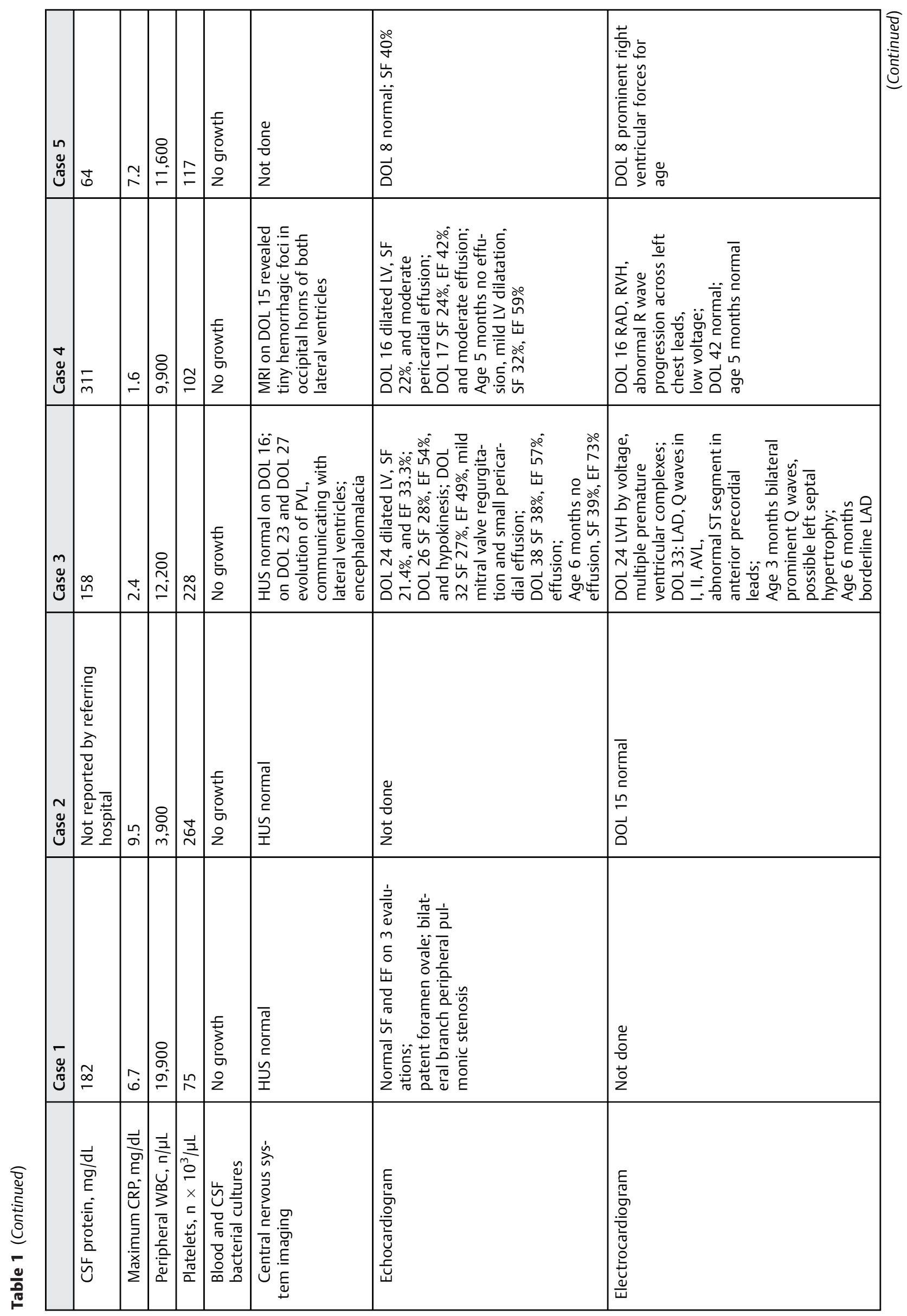


e348 Neonatal Enterovirus Infection Norris et al.

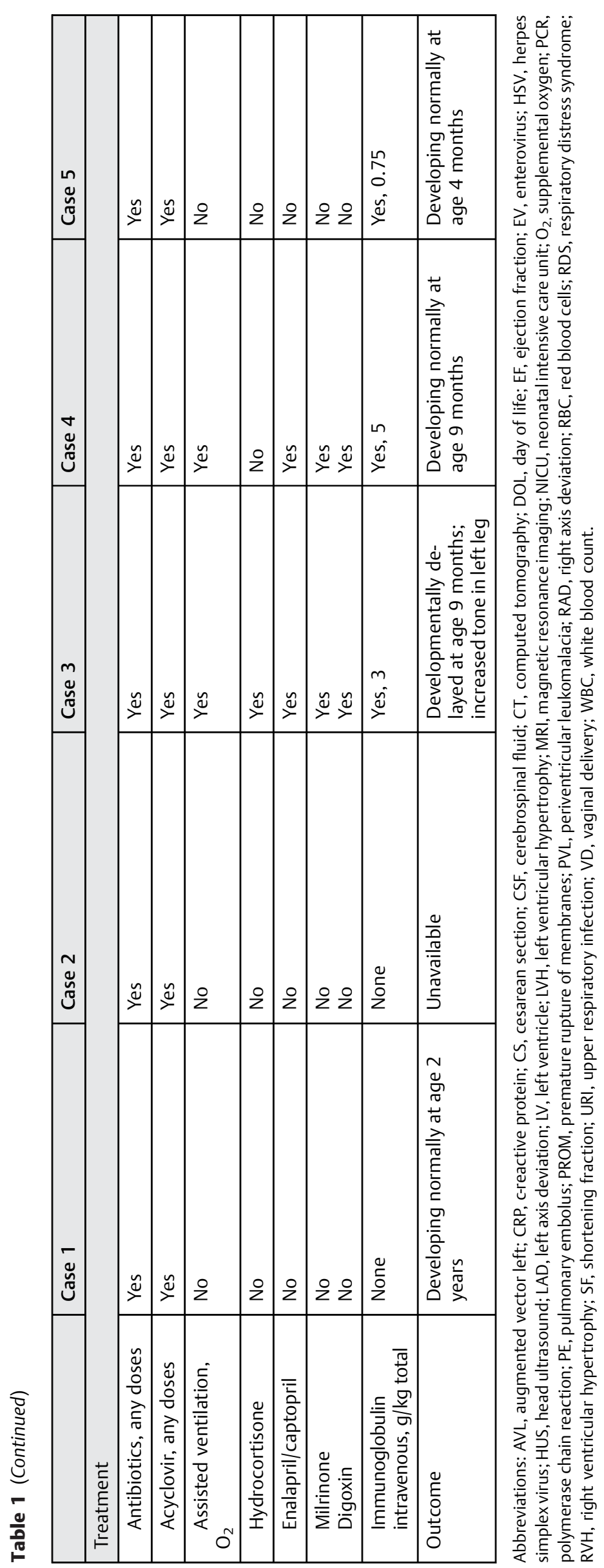

American Journal of Perinatology Reports Vol. 6 No. 3/2016 
PCR analysis of serum samples from 139 consecutive neonates who were admitted during 3 years to hospitals in Kuwait for sepsis, but whose blood cultures were negative for bacteria, identified EV ribonucleic acid in 34 (24\%). ${ }^{14}$

Yet, in a retrospective analysis of clinical practice over 12 years, only 51 of 5,396 (1\%) Dutch infants of $<32$ weeks gestational age or $<1,500 \mathrm{~g}$ birth weight who were admitted for suspected sepsis/meningitis were diagnosed with a viral infection by viral culture and/or PCR test that had been obtained at the discretion of caregivers. ${ }^{15}$ Of these, 20 (39\%) were infections associated with EV or human parechovirus and 2 (4\%) were HSV infections. In the United States, a retrospective analysis of culture-proven etiologic agents in neonatal meningitis at a single institution from 1974 to 1988 identified EV in $10 \%$ overall, with increasing incidence over the period. ${ }^{16}$

EV infections are not reportable nationally, and the current incidence of EV illness among neonates is unknown, but a minimum frequency can be estimated from two sources. First, the National Enterovirus Surveillance System, a voluntary, passive reporting system maintained by the Centers for Disease Control and Prevention, monitors trends in circulating EV identified in CSF, respiratory, and/or fecal specimens by various methods and reported. ${ }^{17}$ The reports reflect a summer-fall seasonality, with $78 \%$ occurring between June and October, inclusive. ${ }^{18}$ Of 22,348 reports of nonpolio EV and human parechovirus detection during 1983 to 2003 that included age of patient ( $84 \%$ of total), 2,544 (11\%) occurred in neonates. ${ }^{19}$ That estimates an average of approximately 120 to $125 \mathrm{EV}$ and human parechovirus laboratory specimen identifications per year among neonates.

The second data source, the 2009 Kids' Inpatient Database survey database of pediatric hospitalizations in the United States, permits estimation of the annual number of hospital diagnoses of neonatal EV central nervous system (CNS) or myocardial infection in the United States ${ }^{20}$ A weighted analysis of the database estimates that 7,469 hospitalized neonates had a primary or secondary diagnosis of a viral infection. Aseptic meningitis due to specified nonbacterial agents was diagnosed in an estimated 477 neonates, and aseptic meningitis due to unspecified nonbacterial agents, which would include EV, was diagnosed in an estimated 952 neonatal patients. The survey frequency of cases of aseptic meningitis attributed to Coxsackie or echo viruses is too low $(<10)$ to estimate the weighted number. Encephalitis due to specified cause, including viruses, was diagnosed in an estimated 26 neonates. Myocarditis caused by Coxsackievirus was estimated to have occurred in 29 neonates, and another 26 were estimated to have been diagnosed with viral or other specified acute myocarditis.

HSV infections may present as neonatal sepsis and/or meningitis, and the incidence has been estimated at 1,500 cases/year. ${ }^{21}$ Neonatologists have a heightened awareness of HSV and routinely obtain CSF for HSV PCR testing in neonates with suspected sepsis and/or meningitis, but they less frequently diagnose or exclude EV infection using a CSF molecular test during the EV season, although the annual incidence of serious, but undiagnosed, EV infection is likely greater than the incidence of HSV infection. 8,22
Diagnosis and illness spectrum: Diagnosing neonatal EV infection is important for several reasons, including avoidance of inappropriate antibiotic therapy; the imperative to detect the complications of myocarditis, hepatitis, and periventricular encephalomalacia; and for infection control. A rapid PCR test for EV in CSF samples makes identification of EV timely. ${ }^{13}$ The test has a sensitivity of $95 \%$, specificity of $100 \%$, positive predictive value of $100 \%$, and negative predictive value of $98 \%$. The test does not distinguish among the serotypes of EV. It also does not detect nucleic acid from human parechovirus type 3 , which may present similarly in the neonatal period and for which there is a different PCR test. $^{23}$ The EV PCR test has become the gold standard for diagnosing EV CNS infections. ${ }^{24,25}$ Cultures of blood and CSF identify EV, but the delay in identification does not help acute management. PCR of blood may be performed, but yields positive results less frequently than does PCR of CSF in young infants with EV infection. ${ }^{26}$

Neonatal EV infections may be acquired from an infected mother, another contact, or as a nosocomial infection. 5,18,27 The illness spectrum in neonates includes a range of severity from inapparent to an acute sepsis-like syndrome, meningitis or meningoencephalitis, and hepatitis. ${ }^{5}$ There is usually a paucity of abnormal CSF findings in EV neonatal sepsis and/or meningitis, often including no pleocytosis or hypoglycemia, and there may be no peripheral blood abnormality except for elevated C-reactive protein and thrombocytopenia, and less often neutropenia. ${ }^{25,28}$

There is no proven antiviral therapy for EV infection. A multicenter randomized placebo-controlled trial of pleconaril (VP63843, ViroPharma, Inc., Exton, PA) for treatment of infants with enterovirus meningitis that was prematurely terminated precluded demonstration of efficacy. ${ }^{29}$ More recently, enrollment has been completed in a phase 2 , multicenter, randomized, placebo-controlled trial of pleconaril for EV sepsis syndrome with severe hepatic involvement, myocardial involvement, and/ or coagulopathy, but results have not been published. ${ }^{30}$ In case reports of older patients with chronic EV meningoencephalitis who were concurrently treated with IVIG, pleconaril effectiveness has been associated with in vitro susceptibility of clinical isolates. ${ }^{31}$ Other candidate antiviral therapies against EV have not been tested in neonates. ${ }^{32}$

Myocarditis IVIG treatment: In two of the five patients in this series myocarditis developed; in one it was not clinically apparent, but was diagnosed by echocardiographic screening. The prognosis for neonates with EV myocarditis is poor: 31 to $83 \%$ die, and among survivors 66\% develop severe cardiac damage. 6,33 Following aggressive supportive treatment that included large doses of IVIG, the myocarditis in our patients resolved. One patient was treated with IVIG in an attempt to prevent myocarditis and did not develop it.

There has been a randomized controlled trial of immunoglobulin therapy with $0.75 \mathrm{~g} / \mathrm{kg}$ in nine neonates with early EV infections, which found no effect on clinical outcomes, ${ }^{34}$ and there is a case report of an infant with Coxsackievirus B1 meningitis who was treated with $0.45 \mathrm{~g} / \mathrm{kg}$ IVIG, but subsequently developed myocarditis. ${ }^{35}$ However, there has been no randomized controlled trial of IVIG therapy of EV in neonates 
with myocarditis. Case reports of unspecified doses of IVIG therapy for EV myocarditis in two neonates have shown inconclusive outcomes. ${ }^{33}$ One case report of IVIG $2 \mathrm{~g} / \mathrm{kg}$ treatment of human parechovirus myocarditis in a 5-month old infant had a favorable outcome. ${ }^{36}$ The effectiveness of IVIG treatment in reducing viremia is a function of the titer of EV type-specific neutralizing antibodies in the IVIG preparation for the infecting EV type; larger doses of IVIG may compensate for lower titers when type-specific neutralizing antibodies are present, but at relatively low titers. ${ }^{31}$ The two patients with neonatal EV myocarditis we report represent successful therapy with IVIG 3 to $5 \mathrm{~g} / \mathrm{kg}$ total dose.

Meningoencephalitis complications: Patient 3 had no documented cerebral hypoxia or hypoperfusion, but developed PVL that progressed to encephalomalacia. PVL or more extensive cerebral white matter injury has been reported as a complication of EV meningoencephalitis in at least eight patients, ${ }^{37-42}$ as well as in at least 11 neonatal parechovirus infections, ${ }^{39,43}$ which is clinically indistinguishable from neonatal EV sepsis and/or meningitis. ${ }^{44}$

\section{Conclusion}

Neonates who appear septic without bacterial etiology during EV seasons may have EV sepsis and/or meningitis that can be diagnosed rapidly by CSF PCR testing. Cases may be underdiagnosed in the early neonatal period if specific testing is not performed. In the absence of EV PCR testing or cultures, the lack of CSF pleocytosis in the EV-infected neonate may lead physicians to fail to make a correct diagnosis and subsequently not screen for serious complications. Patients should be followed for periventricular leukomalacia, signs of myocarditis, and considered for treatment with large total doses of IVIG.

\section{Acknowledgments}

None.

\section{References}

1 Cohen-Wolkowiez M, Moran C, Benjamin DK, et al. Early and late onset sepsis in late preterm infants. Pediatr Infect Dis J 2009; 28(12):1052-1056

2 Hornik CP, Fort P, Clark RH, et al. Early and late onset sepsis in verylow-birth-weight infants from a large group of neonatal intensive care units. Early Hum Dev 2012;88(Suppl 2):S69-S74

3 Rosenlew M, Stenvik M, Roivainen M, Järvenpää AL, Hovi T. A population-based prospective survey of newborn infants with suspected systemic infection: occurrence of sporadic enterovirus and adenovirus infections. J Clin Virol 1999;12(3):211-219

4 Knowles NJ, Hovi T, Hyypiä T, et al. Picornaviridae. In: King AMQ Adams MJ, Carstens EB, Lefkowitz EJ eds. Virus Taxonomy: Classification and Nomenclature of Viruses: Ninth Report of the International Committee on Taxonomy of Viruses. San Diego, CA: Elsevier; 2012:855-880

5 Hawkes MT, Vaudry W. Nonpolio enterovirus infection in the neonate and young infant. Paediatr Child Health 2005;10(7):383-388

6 Freund MW, Kleinveld G, Krediet TG, van Loon AM, VerboonMaciolek MA. Prognosis for neonates with enterovirus myocarditis. Arch Dis Child Fetal Neonatal Ed 2010;95(3):F206-F212
7 Lake AM, Lauer BA, Clark JC, Wesenberg RL, McIntosh K. Enterovirus infections in neonates. J Pediatr 1976;89(5):787-791

8 Jenista JA, Powell KR, Menegus MA. Epidemiology of neonatal enterovirus infection. J Pediatr 1984;104(5):685-690

9 Abzug MJ, Levin MJ, Rotbart HA. Profile of enterovirus disease in the first two weeks of life. Pediatr Infect Dis J 1993;12(10): 820-824

10 Jenista JA, Menegus MA. Neonatal enterovirus infection. Indian J Pediatr 1988;55(2):191-196

11 Abzug MJ. Presentation, diagnosis, and management of enterovirus infections in neonates. Paediatr Drugs 2004;6(1):1-10

12 Tebruegge M, Curtis N. Enterovirus infections in neonates. Semin Fetal Neonatal Med 2009;14(4):222-227

13 Kost CB, Rogers B, Oberste MS, et al. Multicenter beta trial of the GeneXpert enterovirus assay. J Clin Microbiol 2007;45(4): 1081-1086

14 Ahmad S, Dalwai A, Al-Nakib W. Frequency of enterovirus detection in blood samples of neonates admitted to hospital with sepsis-like illness in Kuwait. J Med Virol 2013;85(7):1280-1285

15 Verboon-Maciolek MA, Krediet TG, Gerards LJ, Fleer A, van Loon TM. Clinical and epidemiologic characteristics of viral infections in a neonatal intensive care unit during a 12-year period. Pediatr Infect Dis J 2005;24(10):901-904

16 Shattuck KE, Chonmaitree T. The changing spectrum of neonatal meningitis over a fifteen-year period. Clin Pediatr (Phila) 1992; 31(3):130-136

17 Non-Polio Enterovirus. Outbreaks and Surveillance. Centers for Disease Control and Prevention. Available at: http://www.cdc.gov/non-polioenterovirus/outbreaks-surveillance.html. Accessed February 19, 2013

18 Khetsuriani N, Lamonte-Fowlkes A, Oberste S, Pallansch MA; Centers for Disease Control and Prevention. Enterovirus surveillance-United States, 1970-2005. MMWR Surveill Summ 2006; 55(8):1-20

19 Khetsuriani N, Lamonte A, Oberste MS, Pallansch M. Neonatal enterovirus infections reported to the national enterovirus surveillance system in the United States, 1983-2003. Pediatr Infect Dis J 2006;25(10):889-893

20 Agency for Healthcare Research and Quality. . 2011. Overview of the Kids' Inpatient Database (KID). Available at: http://www. hcup-us.ahrq.gov/kidoverview.jsp. Accessed December 13, 2013

21 Kimberlin DW. Neonatal herpes simplex infection. Clin Microbiol Rev 2004;17(1):1-13

22 Harvala H, McLeish N, Kondracka J, et al. Comparison of human parechovirus and enterovirus detection frequencies in cerebrospinal fluid samples collected over a 5-year period in Edinburgh: $\mathrm{HPeV}$ type 3 identified as the most common picornavirus type. J Med Virol 2011;83(5):889-896

23 Harvala H, Wolthers KC, Simmonds P. Parechoviruses in children: understanding a new infection. Curr Opin Infect Dis 2010;23(3): 224-230

24 Nolte FS, Rogers BB, Tang YW, et al. Evaluation of a rapid and completely automated real-time reverse transcriptase PCR assay for diagnosis of enteroviral meningitis. J Clin Microbiol 2011; 49(2):528-533

25 de Crom SC, van Furth MA, Peeters MF, Rossen JW, Obihara CC. Characteristics of pediatric patients with enterovirus meningitis and no cerebral fluid pleocytosis. Eur J Pediatr 2012;171(5): 795-800

26 Rittichier KR, Bryan PA, Bassett KE, et al. Diagnosis and outcomes of enterovirus infections in young infants. Pediatr Infect Dis J 2005; 24(6):546-550

27 Civardi E, Tzialla C, Baldanti F, Strocchio L, Manzoni P, Stronati M. Viral outbreaks in neonatal intensive care units: what we do not know. Am J Infect Control 2013;41(10):854-856

28 Yun KW, Choi EH, Cheon DS, et al. Enteroviral meningitis without pleocytosis in children. Arch Dis Child 2012;97(10):874-878

29 Abzug MJ, Cloud G, Bradley J, et al; National Institute of Allergy and Infectious Diseases Collaborative Antiviral Study Group. 
Double blind placebo-controlled trial of pleconaril in infants with enterovirus meningitis. Pediatr Infect Dis J 2003;22(4): 335-341

30 Pleconaril Enteroviral Sepsis Syndrome. . Clinical Trials.gov. Available at: http://clinicaltrials.gov/show/NCT00031512. Accessed February 19, 2013

31 Wildenbeest JG, van den Broek PJ, Benschop KSM, et al. Pleconaril revisited: clinical course of chronic enteroviral meningoencephalitis after treatment correlates with in vitro susceptibility. Antivir Ther 2012;17(3):459-466

32 Abzug MJ. The enteroviruses: problems in need of treatments. J Infect 2014;68(Suppl 1):S108-S114

33 Schlapbach LJ, Ersch J, Balmer C, et al. Enteroviral myocarditis in neonates. J Paediatr Child Health 2013;49(9):E451-E454

34 Abzug MJ, Keyserling HL, Lee ML, Levin MJ, Rotbart HA. Neonatal enterovirus infection: virology, serology, and effects of intravenous immune globulin. Clin Infect Dis 1995;20(5): 1201-1206

35 Bae EY, Lee EJ, Han SB, Lee JY, Jeong DC, Kang JH. A case of myocarditis following neonatal meningitis caused by coxsackievirus B1 in spite of intravenous immunoglobulin treatment. J Trop Pediatr 2014;60(2):164-167

36 Wildenbeest JG, Wolthers KC, Straver B, Pajkrt D. Successful IVIG treatment of human parechovirus-associated dilated cardiomyopathy in an infant. Pediatrics 2013;132(1):e243-e247
37 Haddad J, Messer J, Gut JP, Chaigne D, Christmann D, Willard D. Neonatal echovirus encephalitis with white matter necrosis. Neuropediatrics 1990;21(4):215-217

38 Hirata O, Ishikawa N, Mizoguchi Y, Nakamura K, Kobayashi M. A case of neonatal coxsackie B2 meningo-encephalitis in which serial magnetic resonance imaging findings reveal the development of lesions. Neuropediatrics 2011;42(4):156-158

39 Verboon-Maciolek MA, Groenendaal F, Cowan F, Govaert P, van Loon AM, de Vries LS. White matter damage in neonatal enterovirus meningoencephalitis. Neurology 2006;66(8):1267-1269

40 Callen J, Paes BA. A case report of a premature infant with coxsackie B1 meningitis. Adv Neonatal Care 2007;7(5):238-247

41 Hunt JC, Schneider C, Menticoglou S, Herath J, Del Bigio MR. Antenatal and postnatal diagnosis of coxsackie b4 infection: case series. AJP Rep 2012;2(1):1-6

42 van den Berg-van de Glind GJ, de Vries JJ, Wolthers KC, et al. A fatal course of neonatal meningo-encephalitis. J Clin Virol 2012;55(2): 91-94

43 Verboon-Maciolek MA, Groenendaal F, Hahn CD, et al. Human parechovirus causes encephalitis with white matter injury in neonates. Ann Neurol 2008;64(3):266-273

44 Verboon-Maciolek MA, Krediet TG, Gerards LJ, de Vries LS, Groenendaal F, van Loon AM. Severe neonatal parechovirus infection and similarity with enterovirus infection. Pediatr Infect Dis J 2008; 27(3):241-245 